\title{
Determinants of the cytotoxicity of PrrC anticodon nuclease and its amelioration by tRNA repair
}

\author{
BIRTHE MEINEKE and STEWART SHUMAN ${ }^{\mathbf{1}}$ \\ Molecular Biology Program, Sloan-Kettering Institute, New York, New York 10065, USA
}

\begin{abstract}
Breakage of tRNA ${ }^{\mathrm{Lys}(\mathrm{UUU})}$ by the Escherichia coli anticodon nuclease PrrC (EcoPrrC) underlies a host antiviral response to phage T4 infection that is ultimately thwarted by a virus-encoded RNA repair system. PrrC homologs are prevalent in other bacteria, but their activities and substrates are not defined. We find that induced expression of EcoPrrC is toxic in Saccharomyces cerevisiae and E. coli, whereas the Neisseria meningitidis PrrC (NmePrrC) is not. PrrCs consist of an $\mathrm{N}$-terminal NTPase module and a C-terminal nuclease module. Domain swaps identified the EcoPrrC nuclease domain as decisive for toxicity when linked to either the Eco or Nme NTPase. Indeed, a single arginine-to-tryptophan change in the NmePrrC nuclease domain (R316W) educed a gain-of-function and rendered NmePrrC toxic to yeast, with genetic evidence for tRNA ${ }^{\text {Lys(UUU) }}$ being the relevant target. The reciprocal Trp-to-Arg change in EcoPrrC (W335R) abolished its toxicity. Further mutagenesis of the EcoPrrC nuclease domain highlighted an ensemble of 15 essential residues and distinguished between hypomorphic alleles and potential nuclease-nulls. We report that the RNA repair phase of the bacterial virus-host dynamic is also portable to yeast, where coexpression of the T4 enzymes Pnkp and Rnl1 ameliorated the toxicity of NmePrrCR316W. Plant tRNA ligase AtRNL also countered NmePrrC-R316W toxicity, in a manner that depended on AtRNL's 5'-kinase and ligase functions.
\end{abstract}

Keywords: RNA ligase; polynucleotide kinase-phosphatase; ribotoxin; tRNA breakage; wobble uridine modification

\section{INTRODUCTION}

The Escherichia coli tRNA anticodon nuclease PrrC (EcoPrrC) mediates an RNA-damaging innate immune response to bacteriophage T4 infection (Kaufmann 2000). The normally latent EcoPrrC nuclease is switched on by the virus-encoded Stp peptide synthesized early during T4 infection (Amitsur et al. 1989, 1992; Penner et al. 1995). The activated form of EcoPrrC incises the tRNA ${ }^{\text {Lys(UUU) }}$ anticodon loop at a single site $5^{\prime}$ of the wobble uridine, leaving $2^{\prime}, 3^{\prime}$ cyclic phosphate and $5^{\prime}-\mathrm{OH}$ ends at the break. Ensuing depletion of functional tRNA ${ }^{\text {Lys }}$ blocks the synthesis of T4 late proteins and prevents spread of the virus through the bacterial population. However, phage T4 thwarts the host cell's defense strategy by encoding a tRNA repair system, consisting of polynucleotide kinase-phosphatase (Pnkp) and RNA ligase 1 (Rnl1), that heals and seals the broken tRNA ends (Amitsur et al. 1987).

\footnotetext{
${ }^{1}$ Corresponding author.

E-mail s-shuman@ski.mskcc.org.

Article published online ahead of print. Article and publication date are at http://www.rnajournal.org/cgi/doi/10.1261/rna.030171.111.
}

EcoPrrC consists of two domains: an N-terminal nucleoside triphosphate phosphohydrolase (NTPase) module (aa 1-264) related to the ABC transporter NTPase family and a distinctive C-terminal ribonuclease module (aa 265-396) that has no apparent similarity to any known nuclease or tRNA binding protein (Kaufmann 2000; Blanga-Kanfi et al. 2006). PrrC homologs are present in the proteomes of many other bacteria, though their biological activities and RNA targets are uncharted. We reported recently that the ribotoxicity of bacterial PrrC is portable to eukarya. Specifically, we found that induced expression of EcoPrrC in budding yeast cells is fungicidal, signifying that EcoPrrC is toxic in a eukaryon in the absence of any other bacterial or viral proteins (Meineke et al. 2011). Testing for rescue of toxicity by increased tRNA gene dosage implicated tRNA $^{\text {Lys(UUU) }}$ as an EcoPrrC target in yeast. An extensive survey of the effects of alanine and conservative mutations on EcoPrrC toxicity in yeast identified 22 essential residues in the NTPase domain and 11 in the nuclease domain and delineated structure-function relationships at each essential position (Meineke et al. 2011). Overexpressing PrrCs with inactivating mutations in the NTPase active site ameliorated the toxicity of wild-type EcoPrrC; these dominant 
negative effects were not observed with PrrCs containing inactivating mutations in the nuclease domain. Our findings, building on the elegant studies of EcoPrrC by Gabi Kaufmann and colleagues (Meidler et al. 1999; Jiang et al. 2001, 2002; Amitsur et al. 2003; Blanga-Kanfi et al. 2006), support a model in which EcoPrrC toxicity is contingent on head-to-tail dimerization of the ABC-like NTPase domains and the consequent formation of two composite NTP phosphohydrolase active sites, which in turn activates the nuclease domains in cis, only one of which needs be functional.

Remarkably, not all bacterial PrrCs are created equal with respect to their activity in yeast, e.g., Streptococcus mutans PrrC (SmuPrrC) is toxic in yeast, whereas Neisseria meningitidis (NmePrrC) is benign (Meineke et al. 2011). The failure of $N m e P r r C$ to arrest yeast growth was surprising to us, insofar as the nontoxic NmePrrC protein has a significantly higher degree of amino acid identity (57\%) with the EcoPrrC polypeptide than does the toxic SmuPrrC $(42 \%)$. It is conceivable that (1) NmePrrC is nontoxic in yeast because it lacks RNase activity; (2) NmePrrC is a bonafide ribotoxin, but its target is not present in budding yeast (or is present but not essential for yeast growth); or (3) NmePrrC requires additional proteins (or activating metabolites) from the cognate bacterium to manifest its RNase functions.

In the present study, we explored this issue by studying a series of chimeric PrrCs. Domain swaps established that the EcoPrrC nuclease module is decisive for yeast toxicity when linked to either the Eco or Nme NTPase domain. Inspection of the primary structure differences between the nuclease domains of toxic (Eco and Smu) and nontoxic (Nme) PrrCs highlighted potential candidate toxicity determinants that we queried by mutating individual side chains in NmePrrC to their counterparts in EcoPrrC. We thereby identified a single amino acid change in NmePrrC (arginine to tryptophan) that elicited a gain-of-function and rendered it toxic to yeast. The reciprocal Trp to Arg change at the corresponding residue in EcoPrrC ablated its toxicity in yeast. From the results of tRNA rescue experiments and the effects of genetic manipulation of the wobble uridine modification on the activity of the "enabled" NmePrrC-R316W mutant, we surmised that $\mathrm{tRNA}^{\mathrm{Lys}(\mathrm{UUU})}$ is the relevant target for the NmePrrCR316W ribotoxin.

We extended these findings by showing that the RNA repair phase of the bacterial tRNA restriction-repair hostvirus dynamic is also portable to yeast, where coexpression of the T4 enzymes Pnkp and Rnl1 ameliorated the toxicity of NmePrrC-R316W. Expression of plant tRNA ligase in yeast also countered NmePrrC-R316W toxicity. Our findings, in conjunction with earlier studies (Nandakumar et al. 2008), offer proof of principle for the ability of RNA repair to modulate the effects of programmed tRNA damage in eukarya.

\section{RESULTS}

\section{Domain swaps between EcoPrrC and NmePrrC implicate the nuclease domain as the source of species-variation in PrrC toxicity}

The E. coli and N. meningitidis prrC genes were introduced into yeast on CEN plasmids under the control of a glucoserepressed/galactose-inducible GAL1 promoter. EcoPrrC induction inhibited yeast growth on agar medium containing galactose, whereas NmePrrC induction had no effect on cell growth (Fig. 1). An inference from these results is that EcoPrrC can incise essential target RNAs in yeast, but NmePrrC cannot.

Turning to the question of why NmePrrC is nontoxic, we performed reciprocal domain swap experiments, in which the N-terminal NTPase domain of EcoPrrC (aa 1-264) was fused to the C-terminal nuclease domain of NmePrrC (aa 246-380) and the NmePrrC NTPase domain (aa 1-245) was joined to the EcoPrrC nuclease module (aa 265-396) (Fig. 1). The N-Nme/Eco-C chimera was clearly toxic in yeast, while the $\mathrm{N}-E c o / N m e-C$ hybrid was not (Fig. 1). However, the very faint growth of the Nme/EcoPrrCexpressing yeast cells on galactose agar seen with the more concentrated cell spottings (Fig. 1) suggested that the Nmel Eco hybrid is a genetic hypomorph vis à vis EcoPrrC (see below). We conclude that the EcoPrrC nuclease module is decisive for yeast toxicity when linked to either the Eco or Nme NTPase domain.

To see if the differential toxicity of bacterial PrrCs also obtains in a bacterium, we tested the effects of induced expression of the Eco, Smu, and Nme PrrCs and the Eco/ Nme and Nme/Eco chimeras, on the growth of E. coli. The respective prrC genes were introduced into E. coli Top10 cells on pBAD plasmids under the control an arabinoseinducible promoter. Serial dilutions of E. coli pBAD-prrC

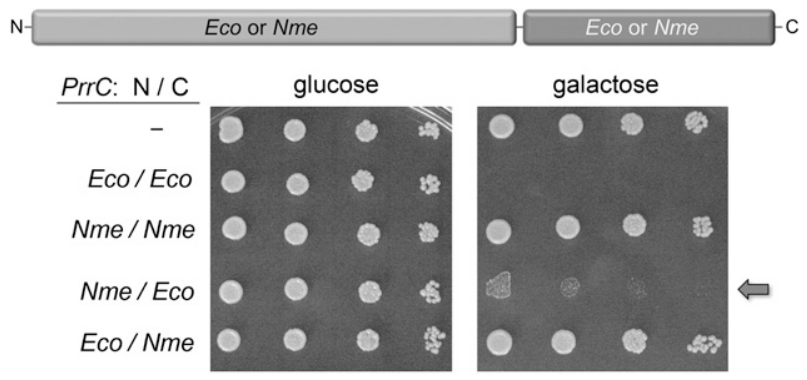

FIGURE 1. Domain swaps between toxic EcoPrrC and nontoxic NmePrrC. Bacterial PrrC proteins consist of an N-terminal NTPase domain fused to a C-terminal nuclease domain, as shown. EcoPrrC and NmePrrC, and domain-swapped PrrCs (N-Eco/Nme-C and N$\mathrm{Nme} / \mathrm{Eco}-\mathrm{C}$ ), were tested for their effects on the growth of S. cerevisiae. Serial fivefold dilutions of yeast cells bearing a CEN plasmid encoding the indicated galactose-regulated $\operatorname{prr} C$ gene or an empty $C E N$ vector $(-)$ were spotted on -Leu agar plates containing glucose or galactose as specified. 
cultures grown in LB medium were plated on LB agar ( $\operatorname{prr} C$ expression repressed) or LB agar with $0.2 \%$ arabinose ( $p r r C$ expression induced). The results showed that EcoPrrC and $S m u$ PrrC were toxic to E. coli, whereas NmePrrC was not (Fig. 2). The Nme/Eco hybrid inhibited growth of E. coli on arabinose agar, albeit not as profoundly as wild-type EcoPrrC, as indicated by the tiny colony size of Nmel EcoPrrC-expressing bacteria (Fig. 2). In contrast, arabinose induction of the Eco/NmePrrC hybrid had no effect on $E$. coli growth (Fig. 2). We surmise from the results shown in Figures 1 and 2 that it is not simply the case that the eukaryal milieu masks an intrinsic ribotoxin activity of NmePrrC and the Eco/Nme hybrid. Rather, it seems that members of the PrrC family differ with respect to their biological activity, which could reflect distinctive RNA target specificities and/or reliance on unique species-specific coactivators. It is pertinent to note that, whereas EcoPrrC targets tRNA ${ }^{\mathrm{Lys}(\mathrm{UUU})}$ in E. coli and yeast, the imputed ribotoxicity and possible RNA targets of NmePrrC are tabula rasa, even in Neisseria.

We next examined the effects of transient expression of the toxic Eco and Smu PrrCs on E. coli survival. The EcoPrrC and SmuPrrC expression plasmids had no effect on the rate of bacterial growth in liquid medium lacking arabinose, i.e., compared to the growth of control bacteria carrying the empty vector (Supplemental Fig. S1A). In contrast, the growth of bacteria carrying the Eco and Smu PrrC plasmids was arrested by 3 to $4 \mathrm{~h}$ after transfer to arabinose-containing medium, an effect not seen with bacteria carrying the empty vector (Supplemental Fig. S1A). By analyzing bacterial survival after transient arabinose induction in liquid medium and return to control medium, we found that EcoPrrC expression was bacteriostatic, i.e., the number of viable bacteria in the culture was

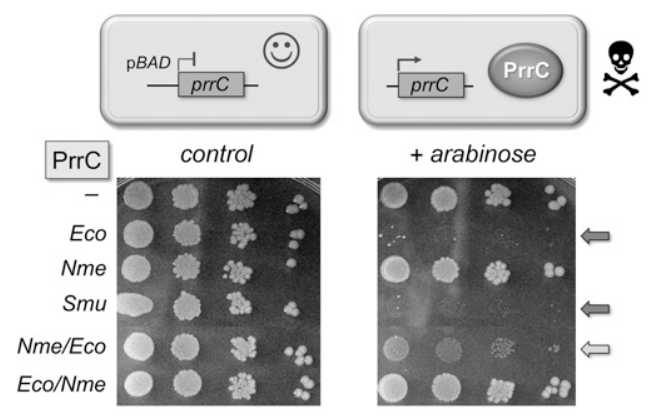

FIGURE 2. The nuclease domain is an exchangeable determinant of PrrC toxicity in E. coli. Serial dilutions of E. coli cells bearing a pBAD plasmid encoding the indicated arabinose-regulated prrC gene or an empty vector were spotted on LB-ampicillin agar plates with or without arabinose. When grown on control medium lacking arabinose, $\operatorname{prr} C$ expression is switched off, and the bacteria grow normally. When grown on medium containing $0.2 \%$ arabinose, the prrC expression is turned on, and if the PrrC ribotoxin is active, bacterial growth is arrested (absence of colonies) or slowed (presence of tiny colonies). stable for the 5-h interval of arabinose exposure. (Supplemental Fig. S1B). In contrast, SmuPrrC was bactericidal, eliciting a 240 -fold decrement in the viable cell count by $3 \mathrm{~h}$ of SmuPrrC induction (Supplemental Fig. S1B). We noted progressive recovery of viability at 4 and $5 \mathrm{~h}$ post-induction of $S m u$ PrrC, suggesting the outgrowth of survivors. The instructive point here is that the cytostatic effect of EcoPrrC expression in E. coli contrasts with its cytocidal properties in budding yeast (Meineke et al. 2011), as opposed to SmuPrC expression, which is cytocidal in bacteria and yeast.

\section{Species-specific toxicity determinants in the PrrC nuclease domain}

To vet the hypothesis that the PrrC nuclease domain harbors key determinants of RNA target specificity, we attempted to coax the nontoxic NmePrrC to become toxic in yeast. Our search for gain-of-function mutations was guided by alignment of the nuclease domains of the Eco, Smu, and Nme PrrCs, which highlighted seven "deviant" amino acids in nontoxic NmePrrC that we changed to the "consensus" equivalents present in the toxic EcoPrrC and SmuPrrC proteins, individually or as a pairwise change in vicinal residues (Fig. 3A). We found that five of the NmePrrC mutants (N309S, E322D, Q354K, D366N, and E288A-N289D) remained nontoxic in yeast. In contrast, the NmePrrC R316W mutant was enabled by a single amino acid substitution to arrest the growth of yeast cells on medium containing galactose (Fig. 3B). Thus, a gain-of-function was achieved. In a similar vein, we were able to convert the nontoxic Eco/ Nme PrrC chimera (Fig. 1) into an active ribotoxin in yeast by the equivalent Arg-to-Trp mutation in its Nme-derived nuclease domain (data not shown).

If the identity of this amino acid as tryptophan is decisive as a toxicity determinant in $\operatorname{PrrC}$, then we might expect mutation of Trp335 in EcoPrrC to diminish or eliminate its ribotoxicity. Indeed, changing Trp335 to arginine (to mimic the side chain in NmePrrC) abolished the toxicity of EcoPrrC in yeast (Fig. 3C). Similar loss-of-function effects were elicited by changing Trp335 to alanine (which truncates the side chain at the $\beta$-carbon) or by conservative substitutions with other $\gamma$-branched aromatic amino acids (tyrosine, phenylalanine, or histidine) (Fig. 3C). Thus, tryptophan is strictly essential at this position for the ribotoxicity of $E c o$ PrrC in yeast.

We compared the severity of the yeast growth arrest triggered by the two gain-of-function mutants-NmeR316W and Eco/NmeRW - to that of the active Nme/Eco chimeric PrrC. By analyzing yeast survival after transient galactose induction and return to glucose, we found that Eco/NmeRW expression was fungicidal; the number of viable cells in the yeast culture decreased by a factor of 20 after $15 \mathrm{~h}$ of induction (Supplemental Fig. S2). In contrast, expression of the NmeR316W and Nme/Eco PrrC proteins was effectively 
A

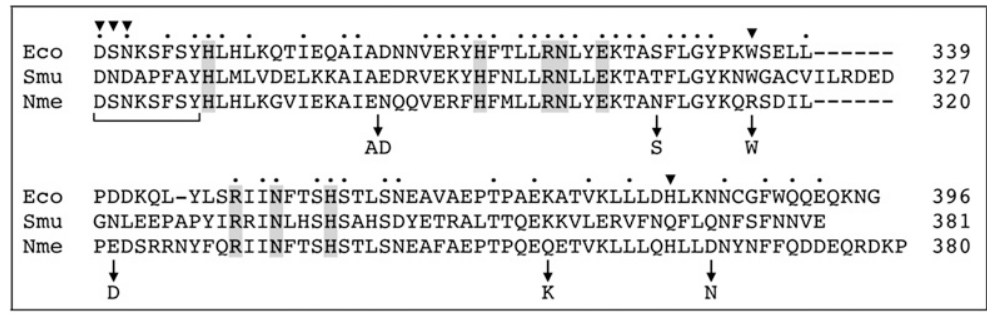

B

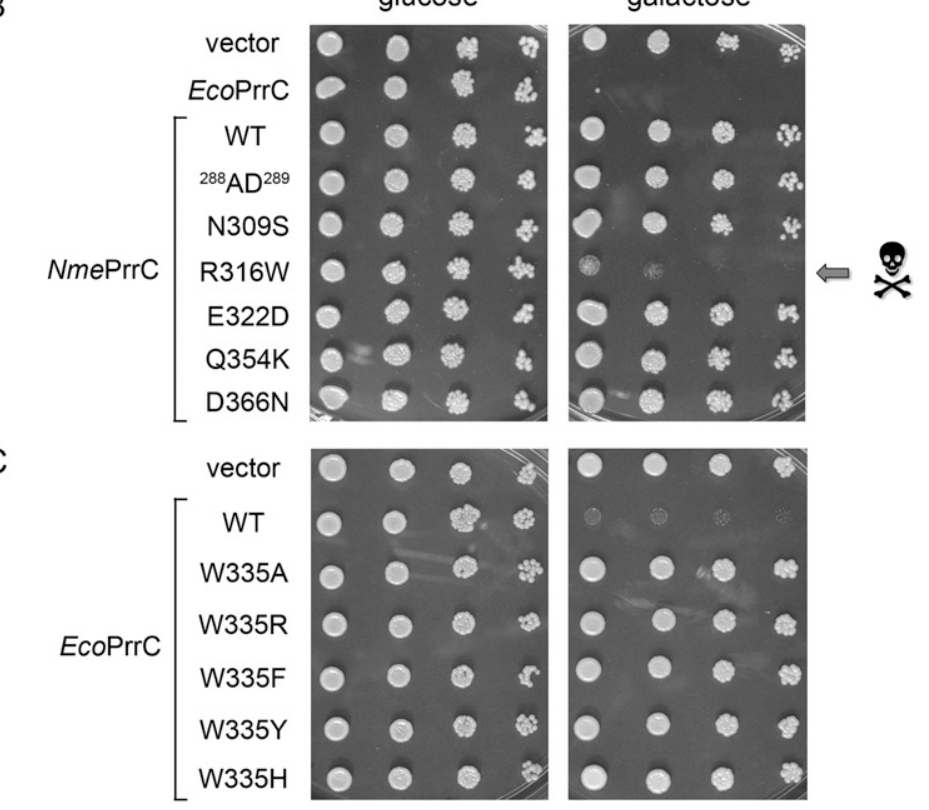

FIGURE 3. A gain-of-function mutation renders NmePrrC toxic to yeast. (A) The amino acid sequence of the nuclease domain of EcoPrrC is aligned to the homologous segments of SmuPrrC and NmePrrC. Positions of side-chain identity/similarity in all three proteins are indicated by $\bullet$ above the alignment. The eight conserved residues defined previously as essential for yeast toxicity are shaded gray. Positions of side chain variation between the nontoxic $\mathrm{NmePrC}$ and the toxic Eco and Smu PrrCs are indicated by arrows below the alignment, which specify the NmePrrC mutations tested for gain-of-toxicity in yeast. The LARP motif is demarcated by the bracket below the sequences. EcoPrrC residues subjected to mutational analysis in the present study are indicated by $\boldsymbol{\nabla}$. (B) Serial fivefold dilutions of yeast cells bearing a $C E N$ plasmid encoding the indicated galactose-regulated $\operatorname{prr} C$ gene or an empty $C E N$ vector were spotted on -Leu agar plates containing glucose or galactose as specified. $(C)$ Toxicity tests for wild-type EcoPrrC and the indicated W335 mutants are shown.

cytostatic, i.e., viable cell counts increased less than threefold over $15 \mathrm{~h}$ in galactose-containing medium (Supplemental Fig. S2).

\section{Overexpression of yeast tRNA ${ }^{\text {Lys(UUU) }}$ blunts the toxicity of NmeR316W}

If an intracellular ribotoxin exerts its effect by breaking a specific cellular RNA target, then one might expect to reverse the toxicity by overexpressing the RNA target (Jablonowski et al. 2006). We found that a multicopy $2 \mu$ plasmid carrying the yeast gene for $\mathrm{TRNA}^{\mathrm{Lys}(\mathrm{UUU})}$ protected yeast cells from the toxic effects of the gain-of-function mutant NmeR316W (Fig. 4, top panel). In contrast, $2 \mu$ plasmids bearing genes encoding eitherthe isoacceptor tRNA ${ }^{\text {Lys(CUU) }}$ or various other yeast tRNAs $\left(\mathrm{tRNA}^{\mathrm{Glu}} \mathrm{tRNA}^{\mathrm{Gln}}\right.$, $\mathrm{tRNA}^{\mathrm{Arg}}$, $\mathrm{tRNA}^{\mathrm{Tyr}}, \mathrm{tRNA}^{\mathrm{Leu}}$, or tRNA ${ }^{\text {Gly }}$ ) had no effect on NmeR316W toxicity (data not shown). This instructive result indicated that tRNA ${ }^{\text {Lys(UUU) }}$ is a target of NmeR316W in yeast. The same tRNA rescue profile was seen previously for two toxic hypomorphs of EcoPrrC (Meineke et al. 2011). We surmise that a latent tRNA $^{\text {Lys(UUU) }}$ anticodon nuclease activity of NmePrrC is revived by the R316W mutation.

\section{Influence of wobble uridine modifications on the toxicity of NmeR316W}

EcoPrrC incises bacterial tRNA ${ }^{\text {Lys(UUU) }}$ at a single phosphodiester $5^{\prime}$ of the modified wobble base $\mathrm{mnm}^{5} \mathrm{~s}^{2} \mathrm{U}$ (5-methylaminomethyl-2-thiouridine) (Jiang et al. 2001). The $\mathrm{mnm}^{5} \mathrm{U}$ wobble modification does not exist in eukaryal tRNAs, which have $\mathrm{mcm}^{5} \mathrm{~s}^{2} \mathrm{U}$ (5-methoxycarbonylmethyl-2-thiouridine) instead (Fig. 4). Some tRNA anticodon nucleases rely on the modified wobble base as a target specificity determinant. For example, $K$. lactis $\gamma$-toxin requires the $\mathrm{mcm}^{5} \mathrm{U}$ modification in its $\mathrm{tRNA}^{\mathrm{Glu}}$ target, such that yeast elp $3 \Delta$ and trm $9 \Delta$ mutants, which either fail to modify the C5 atom or fail to add the terminal methyl group (Fig. 4), are resistant to $\gamma$-toxin's effects ( $\mathrm{Lu}$ et al. 2005, 2008; Jablonowski and Schaffrath 2007). Here we found that $N m e$ R $316 \mathrm{~W}$ was toxic to elp $3 \Delta$ cells and that this toxicity was reversed by overexpressing tRNA ${ }^{\text {Lys(UUU) }}$ (Fig. 4, middle panel), which signifies that NmeR316W can target tRNA with a wobble uridine with no modifications at the $\mathrm{C} 5$ atom. In contrast, NmeR316W did not prevent growth of the trm9s cells on galactose; rather it had only a slight effect on growth, as gauged by colony size compared to the vector control, and this slight effect was reversed by $2 \mu \mathrm{tRNA}^{\text {Lys(UUU) }}$ (Fig. 4, bottom panel). We noted similar effects of the yeast elp $3 \Delta$ and trm $9 \Delta$ mutations on the ribotoxin activities of two EcoPrrC hypomorphs: S219T and C386A (Meineke et al. 2011). Our findings suggest that NmeR316W has gained both the activity and target specificity of EcoPrrC in yeast, albeit at the level of a hypomorphic EcoPrrC variant. 


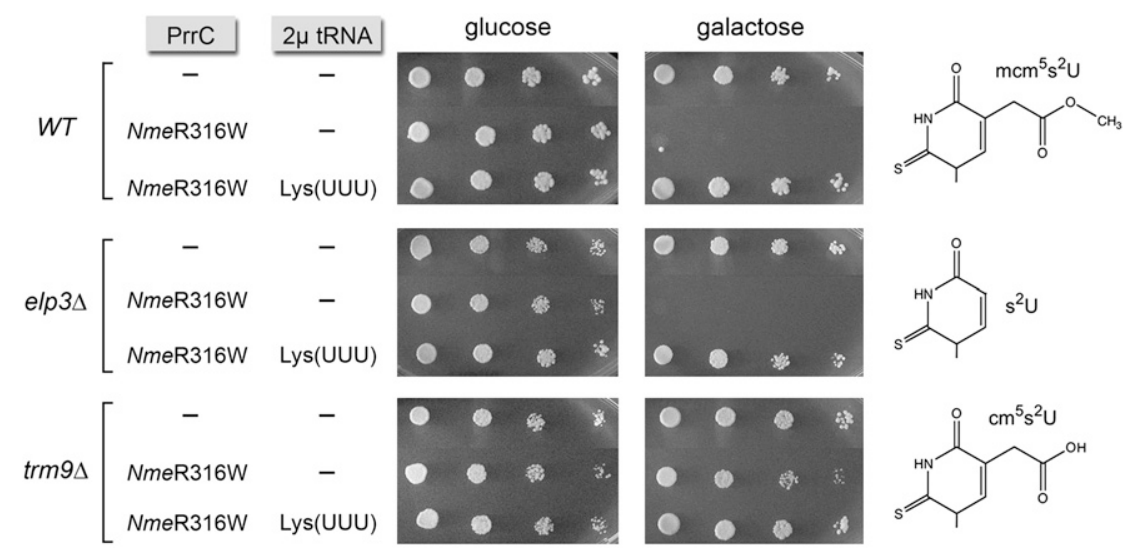

FIGURE 4. Rescue of NmePrrC-R316W toxicity by $2 \mu$ tRNA $^{\text {Lys(UUU) }}$ and effect of wobble U modification on toxicity. Serial fivefold dilutions of wild-type (WT), elp $3 \Delta$, and trm $9 \Delta$ yeast cells bearing a $C E N$ plasmid encoding galactose-regulated $N m e$ PrrC-R316W or the empty CEN vector $(-)$ plus a $2 \mu$ plasmid carrying the tRNA ${ }^{\mathrm{Lys}(\mathrm{UUU})}$ gene or an empty $2 \mu$ vector (-) were spotted on -Leu-Ura agar plates containing glucose or galactose. The structures of the wobble uridine modifications found in tRNA ${ }^{\mathrm{Lys}(\mathrm{UUU})}$ of wild-type yeast $\left(\mathrm{mcm}^{5} \mathrm{~s}^{2} \mathrm{U}\right)$, and yeast mutants elp $3 \Delta$ ( $\mathrm{s}^{2} \mathrm{U} ; 2$-thiouridine) and $\operatorname{trm} 9 \Delta\left(\mathrm{cm}^{5} \mathrm{~s}^{2} \mathrm{U} ; 5\right.$-carboxymethyl-2-thiouridine), are shown at right.

\section{Heterologous RNA repair enzymes ameliorate the toxicity of NmeR316W}

Yeast cells are susceptible to tRNA ribotoxins because the endogenous yeast tRNA ligase is unable to rectify the break in the anticodon loop of the tRNAs targeted by the ribotoxin. However, expression of plant or phage T4 tRNA repair enzymes protect yeast from growth arrest by $K$. lactis $\gamma$-toxin because they are able to reverse the damage inflicted in the anticodon loop of tRNA ${ }^{\mathrm{Glu}(\mathrm{UUC})}$ (Nandakumar et al. 2008). Given that the native function of the T4 RNA repair system is to neutralize the PrrC-driven antiviral response (Amitsur et al. 1987), it was of interest to us to see whether importing phage and plant tRNA repair enzymes into yeast might protect a eukaryon against PrrC's toxicity. Our initial experiments showed that neither plant tRNA ligase (AtRNL) nor T4 Rnl1+Pnkp were able to overcome galactose-induced EcoPrrC growth arrest (data not shown). We considered two potential explanations for the negative outcome: (1) that the heterologous repair enzymes were inherently unable to fix the PrrC-induced damage to yeast tRNA $^{\mathrm{Lys}(\mathrm{UUU})}$; or (2) that the level of tRNA incision activity of EcoPrrC after galactose induction was too vigorous to be offset by the activities of the heterologous repair systems, i.e., the RNA repair system loses an uphill battle against relentless tRNA cleavage. In the latter case, we might expect that dialing back on the strength of the PrrC activity, by expressing a hypomorphic version of the ribotoxin, might tip the dynamic in favor of RNA repair. Indeed, this is what we observed when the NmeR $316 \mathrm{~W}$ variant was induced in yeast cells expressing heterologous repair enzymes (Fig. 5).

In this experiment, we introduced CEN plasmids expressing Rnl1 and Pnkp into a yeast strain bearing the galactose-regulated $N m e \mathrm{R} 316 \mathrm{~W}$ expression plasmid and then tested growth under toxin-off and toxin-on conditions. For comparison, we also tested the effects of overexpressing the yeast tRNA ligase Trl1 (by introducing a $2 \mu$ plasmid bearing TRL1 under the control of a constitutive yeast TPII promoter) and the plant tRNA ligase AtRNL (delivered on a $2 \mu$ plasmid and driven by the TPI1 promoter). Whereas control cells and $2 \mu$ TRL1 cells did not thrive on medium containing galactose, the phage tRNA repair system and plant AtRNL allowed cell growth (Fig. 5). It is apparent from the colony size that the phage tRNA repair system is more salutary than AtRNL and that neither repair system restored growth on galactose to the level of control cells that lack the NmeR316W expression cassette (Fig. 5). This result underscores the theme (Nandakumar et al. 2008) that a repair-based cure of ribotoxicity may be incomplete in the face of constitutive RNA damage.

The observation that plant tRNA ligase rescues cells from NmePrrC-R316W growth arrest while yeast tRNA ligase does not suggests that there are intrinsic differences in the ability of the plant and yeast systems to repair the broken tRNA $^{\text {Lys(UUU) }}$ anticodon loop. To probe the roles of the

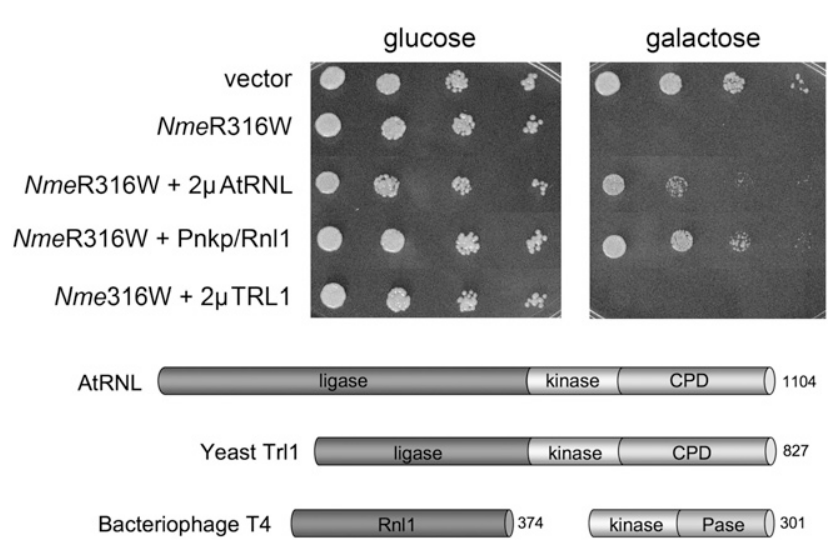

FIGURE 5. Rescue of NmePrrC-R316W toxicity by tRNA repair enzymes. (Top panel) Growth of yeast cells bearing a CEN plasmid encoding galactose-regulated NmePrrC-R316W or the empty CEN vector $(-)$ and either a $2 \mu$ TPI1-AtRNL plasmid, a $2 \mu$ TPI1-TRL1 plasmid, or a CEN plasmid expressing T4 Pnkp and Rnl1 as specified was assessed by spotting serial fivefold dilutions to minimal synthetic agar medium containing glucose or galactose. (Bottom panel) The tRNA ligases of plant (AtRNL) and yeast (Trl1) are composed of three discrete catalytic domains: an N-terminal ligase module; a central $5^{\prime}$ OH polynucleotide kinase module; and a C-terminal RNA 2',3' cyclic phosphodiesterase (CPD) module. The phage T4 tRNA repair system consists of separate sealing (Rnll) and healing (Pnkp) enzymes. 
three catalytic activities of AtRNL, we tested a collection of mutant AtRNL alleles bearing lethal alanine mutations in the active sites that specifically ablate the ligase (K152A or E326A), kinase (S701A or D726A), or CPD (T1001A or H1060A) functions (Fig. 6; Wang et al. 2006). The wildtype, kinase-dead, ligase-dead, and CPD-dead AtRNL proteins were expressed from $2 \mu$ plasmids in yeast cells bearing the NmeR316W plasmid and tested in parallel for growth on glucose and galactose media (Fig. 6). None of the AtRNL-Ala mutations affected growth on glucose because tRNA splicing activity is provided by the endogenous $\operatorname{Trl} 1$ enzyme. However, these mutations had disparate effects on yeast growth on galactose, depending on which catalytic activity was affected. The CPD activity of AtRNL was not required to confer $N m e \mathrm{R} 316 \mathrm{~W}$ resistance, insofar as the CPD-dead alleles could rescue growth on galactose, albeit not as effectively as wild-type AtRNL (Fig. 6). We surmise that the endogenous level of yeast Trll CPD suffices to heal the $2^{\prime}, 3^{\prime}$ cyclic phosphate end of broken tRNA ${ }^{\text {Lys }}$. In contrast, the two ligase-dead and the two kinase-dead AtRNL mutants failed to protect against NmeR316W (Fig. 6). Thus, the ligase and $5^{\prime}$ kinase activities of AtRNL are essential for NmeR316W resistance.

These enzymatic requirements for AtRNL repair of PrrC damage in vivo are different from what was observed for AtRNL rescue of tRNA ${ }^{\text {Glu }}$ damage by $K$. lactis $\gamma$-toxin. Protection against $\gamma$-toxin required the ligase activity but was

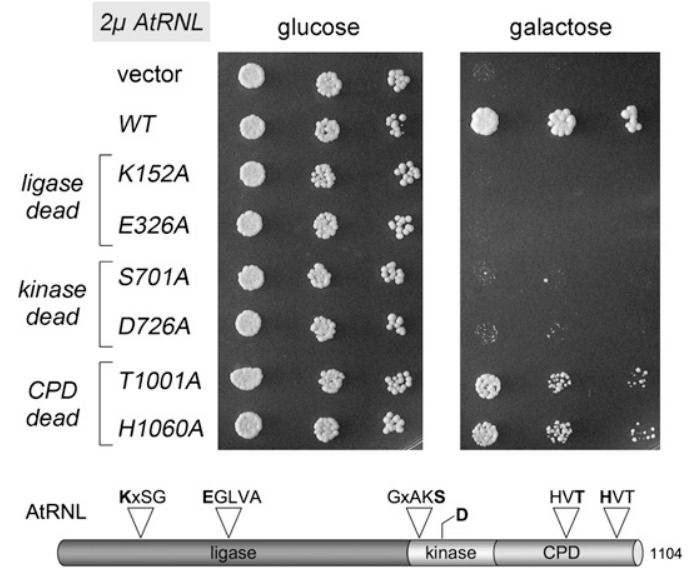

FIGURE 6. RNA sealing and $5^{\prime}$ healing activities of AtRNL dictate resistance to PrrC. (Top panel) Growth of yeast cells bearing a CEN plasmid encoding galactose-regulated NmePrrC-R316W or the empty CEN vector (-) and either an empty $2 \mu$ HIS3 vector or a $2 \mu$ HIS 3 TPI1-AtRNL plasmid encoding wild-type plant tRNA ligase or the indicated ligase-dead, kinase-dead, or CPD-dead mutant was assessed by spotting serial fivefold dilutions to minimal synthetic agar medium containing glucose or galactose. The galactose plate was photographed after $4 \mathrm{~d}$ of incubation at $30^{\circ} \mathrm{C}$. (Bottom panel) AtRNL mutants. The positions of the covalent adenylylation motif $(\mathrm{KxxG})$ and the metalbinding motif (EGxxx) at the ligase active site, the P-loop motif $\operatorname{GxxK}(\mathrm{S} / \mathrm{T})$ at the kinase active site, and the two HxT motifs that comprise the CPD active site are depicted above the AtRNL polypeptide. The sites of enzyme-inactivating alanine mutations in the AtRNL active sites are highlighted in bold. unaffected by the kinase-dead mutations (Nandakumar et al. 2008). We envision that these differences might be attributable to the fact that PrrC and $\gamma$-toxin incise the anticodon loop on opposite sides of the $\mathrm{mcm}^{5} \mathrm{~s}^{2} \mathrm{U}$ wobble nucleoside in their respective tRNA targets. $\gamma$-toxin cleaves on the $3^{\prime}$ side of the wobble nucleoside to form a $\mathrm{mcm}^{5} \mathrm{~s}^{2} \mathrm{U}-2^{\prime}, 3^{\prime}$-cyclic phosphate end that, when hydrolyzed by the CPD activity of Trll or AtRNL, will yield an $\mathrm{mcm}^{5} \mathrm{~s}^{2} \mathrm{U}-3^{\prime} \mathrm{OH}, 2^{\prime}-\mathrm{PO}_{4}$ end. It was proposed that (1) the Trl1 ligase domain is hindered from sealing $\gamma$-toxin-incised tRNA ${ }^{\text {Glu }}$ by the presence of the bulky $\mathrm{mcm}^{5} \mathrm{~s}^{2} \mathrm{U}$ base at the $3^{\prime}-\mathrm{OH}, 2^{\prime}-\mathrm{PO}_{4}$ end; (2) the unmodified $5^{\prime}-\mathrm{OH}$ nucleoside at the $\gamma$-toxin incision site in tRNA ${ }^{\text {Glu }}$ can be phosphorylated by either the Trl1 or AtRNL kinase domains, accounting for the ability of kinase-dead AtRNL to rescue growth on galactose; and (3) the capacity of AtRNL to rectify $\gamma$-toxin damage is a unique property of its ligase domain, which is apparently adept at sealing the broken tRNA with a bulky $\mathrm{mcm}^{5} \mathrm{~s}^{2} \mathrm{U}$ base at the $3^{\prime}-\mathrm{OH}, 2^{\prime}-\mathrm{PO}_{4}$ end (Nandakumar et al. 2008). Extending that line of reasoning to PrrC, which incises on the $5^{\prime}$ side of the wobble nucleoside to yield an unmodified $2^{\prime}, 3^{\prime}$ cyclic phosphate terminus and a $5^{\prime}-\mathrm{OH}$ $\mathrm{mcm}^{5} \mathrm{~s}^{2} \mathrm{U}$ terminus, leads to the following speculations: (1) The Trl1 kinase module is ineffective at phosphorylating the bulky $5^{\prime}-\mathrm{OH} \mathrm{mcm}^{5} \mathrm{~s}^{2} \mathrm{U}$ nucleoside, whereas the AtRNL kinase is competent to do so; and (2) the Trll ligase domain is ineffective at sealing the healed $5^{\prime}-\mathrm{PO}_{4}$ end with a bulky $\mathrm{mcm}^{5} \mathrm{~s}^{2} \mathrm{U}$ nucleoside, whereas the AtRNL ligase is competent to do so. Nothing is known as yet concerning the structural features of the yeast and plant ligases that dictate their differential healing and sealing of base-modified RNA breaks.

\section{Further mutational analysis of the EcoPrrC nuclease domain}

Our previous study of the effects of alanine and conservative mutations on EcoPrrC toxicity in yeast identified 11 essential residues in the C-terminal nuclease domain. Here, we identified $\operatorname{Trp} 335$ as an additional essential constituent of EcoPrrC (Fig. 3C). To extend the structure-function analysis of the nuclease domain, we initiated a new round of alanine and conservative mutagenesis, focusing mainly on a putative lysine anticodon recognizing peptide (LARP) motif, ${ }^{284}{ }^{K Y G D S N K S F S Y}{ }^{294}$, that had been the subject of studies by the Kaufmann lab (Klaiman et al. 2007). The LARP motif (denoted by bracket in Fig. 3A), mutations of which affect the tRNA substrate preference of EcoPrrC (Jiang et al. 2001, 2002), is found only in a subset of PrrC proteins (Klaiman et al. 2007; Davidov and Kaufmann 2008). It is speculated that LARP is a determinant of the target specificity of those PrrC proteins that contain the motif. However, LARP may not be the decisive factor with respect to yeast toxicity of bacterial PrrCs, insofar as the EcoPrrC LARP is not conserved (only $3 / 11$ identical residues) in $S m u P r r C$, which is toxic in yeast. We reported previously 
that two alanine mutations in the EcoPrrC LARP motif (at Ser291 and Ser293, which are conserved in NmePrrC) (Fig. 3A) had no effect on cytotoxicity in yeast (Meineke et al. 2011).

Here we introduced alanine in lieu of EcoPrrC LARP motif residues Asp287, Ser288, and Asn289, and also at the distal residue His381 (Fig. 3A, targeted positions denoted by $\boldsymbol{\nabla})$. The mutant alleles were inserted into CEN plasmids under $G A L$-control. Tests of yeast growth on glucose and galactose showed that the D287A, S288A, and H381A mutants were nontoxic, whereas $\mathrm{N} 289 \mathrm{~A}$ retained toxicity (Fig. 7; and data not shown). Substituting His381 conservatively with glutamine and asparagine also rendered EcoPrrC nontoxic in yeast (data not shown). Thus, His381 joins four other histidines in the nuclease domain (His295, His297, His315, and His356) as strictly essential for EcoPrrC toxicity in yeast (Meineke et al. 2011). Because His381 is replaced by glutamine in the toxic SmuPrrC protein (Fig. 3A), we suspect that His381 is not acting as general acid-base catalyst of RNA transesterification. Replacing the essential LARP motif residue Ser288 with asparagine restored toxicity to EcoPrrC (data not shown); note that asparagine is naturally present at the equivalent position of SmuPrrC. These findings suggest that the hydrogen bonding capacity of Ser288 is pertinent for PrrC activity in yeast.

We were especially interested in structure-activity relations at the essential Asp287, in light of prior suggestions

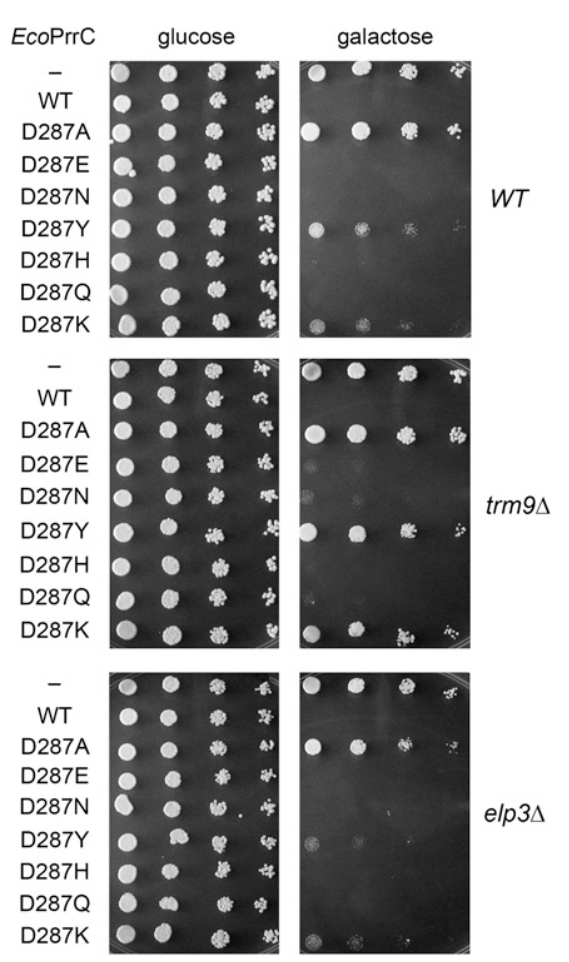

FIGURE 7. Mutational analysis of EcoPrrC Asp287. Serial fivefold dilutions of wild-type (WT), elp $3 \Delta$, and trm9s yeast cells bearing a CEN plasmid encoding galactose-regulated EcoPrrCs as specified were spotted on -Leu agar plates containing glucose or galactose. that this residue is a target specificity determinant (Meidler et al. 1999; Jiang et al. 2001, 2002). Thus, we replaced Asp287 conservatively with glutamate and asparagine and nonconservatively with tyrosine, histidine, glutamine, and lysine (Fig. 7). The D287E, D287N, D287H, and D287Q mutants displayed full galactose-dependent toxicity in yeast (Fig. 7, top panel), suggesting that hydrogen-bonding might be the key property of this side chain. Certainly, the results exclude a strict requirement for negative charge at this position. On the other hand, the D287Y and D287K mutants were partially inhibitory to yeast growth, insofar as the yeast cells expressing these variants grew on galactose agar but formed tiny colonies compared to D287A-expressing cells or the vector control (Fig. 7, top panel). We surmise that charge inversion (in D287K) or increased side chain bulk (in D287Y) at this position exert negative effects on EcoPrrC function in yeast.

The Kaufmann lab had shown that missense mutations at Asp287 alter the tRNA cleavage preferences of PrrC in vivo when expressed in E. coli and/or in vitro, e.g., such that particular Asp287 mutants are either more or less fastidious regarding the impact of wobble base modifications (Jiang et al. 2001, 2002). In this light, we compared the effects of induced expression of Asp287A mutants in yeast cells that have the wild-type $\mathrm{mcm}^{5} \mathrm{~s}^{2} \mathrm{U}$ wobble modification versus elp3s and trm9s cells that lack all or part of the $\mathrm{mcm}^{5}$ moiety (Fig. 7). The salient findings were as follows: (1) PrrC mutants D287Y and D897K were less toxic in trm9s cells than in wild-type or elp3s cells; and (2) other nonalanine Asp287 mutants retained toxicity in all three strain backgrounds (Fig. 7). The findings fortify our inferences from the analysis of NmeR316W toxicity (Fig. 4) that hypomorphic PrrC variants have diminished ability to inflict damage at an incompletely modified $\mathrm{cm}^{5} \mathrm{~s}^{2} \mathrm{U}$ wobble nucleoside.

\section{Restoration of the toxicity of a subset of PrrC nuclease domain mutants by increased gene dosage}

We have now identified a total of 15 residues in the nuclease domain of EcoPrrC that are essential for toxicity in yeast. This module has no discernible primary structure similarity to any known ribonucleases or tRNA-binding proteins, which makes it difficult to guess which essential residues might be directly involved in catalysis versus substrate recognition, PrrC folding/stability, etc. As discussed above, the mutational analysis readily identified PrrC hypomorphs that retained toxicity in yeast when expressed from a CEN plasmid but were either amenable to alleviation of toxicity by co-expression of RNA repair enzymes (unlike wild-type PrC) or were affected in their toxicity by the status of the wobble uridine modification (also unlike wild-type PrrC). Other PrrC hypomorphs (e.g., D287K) were simply less inhibitory to yeast growth than wild-type PrrC when expressed from a CEN plasmid. 
Taking this one step further, we reasoned that (1) the collection of 15 PrrC-Ala mutants in the nuclease domain that were deemed nontoxic when expressed from CEN plasmids might include additional, more severely affected hypomorphs; (2) some of these putative hypomorphs might regain their toxicity when expressed from a multicopy $2 \mu$ plasmid; and (3) recovery of toxicity by overexpression of a particular mutant would weigh against the mutated residue being strictly essential for catalysis.

To evaluate this scenario, we transferred the $15_{G A L}$ - $p r r C$ Ala expression cassettes to $2 \mu$ plasmids, introduced them into Saccharomyces cerevisiae, and tested the transformants for galactose-dependent toxicity (Table 1). Two of the originally nontoxic mutants regained full toxicity at high gene dosage (scored as ++ in Table 1): These were H297A and K299A. Three other mutants-S288A in the LARP motif, N352A, and His381A-regained partial activity (scored as + in Table 1). We surmise that these six side chains are unlikely to be directly catalytic. We presume that the set of nine EcoPrrC-Ala mutants that did not regain toxicity at high gene dosage encompasses bonafide constituents of the nuclease active site. Among the candidate active site residues are three strictly essential and conserved histidines (His295, His315, His356), two strictly essential and conserved arginines (Arg320 and Arg349), and one strictly essential and conserved glutamate (Glu324). The Kaufmann lab had proposed that Arg320, Glu324, and His356 comprise a catalytic triad that they implicate in chemical catalysis of transesterification at the wobble nucleotide to generate $2^{\prime}, 3^{\prime}$ cyclic phosphate and $5^{\prime}-\mathrm{OH}$ product strands (Blanga-Kanfi et al. 2006). Our results are consistent with their model but raise the prospect that additional residues might play a catalytic role. Of course, a definitive interpretation of the mutational data awaits an atomic structure of the nuclease domain.

TABLE 1. High gene dosage can restore toxicity of certain defective PrrC nuclease domain mutants

\begin{tabular}{lc}
\hline $2 \mu$ PrrC & Toxicity on galactose \\
\hline D267A & - \\
D287A & - \\
S288A & + \\
H295A & - \\
H297A & ++ \\
K299A & ++ \\
H315A & - \\
R320A & - \\
N321A & + \\
E324A & - \\
W335A & - \\
R349A & - \\
N352A & - \\
H356A & + \\
H381A & - \\
\hline
\end{tabular}

\section{DISCUSSION}

Programmed tRNA damage by site-specific endoribonucleases is a shared feature of cellular stress responses and self-nonself discrimination in a wide range of prokaryal and eukaryal taxa. tRNA anticodon breakage results in inhibition of protein synthesis, either by depletion of the pool of specific tRNA isoacceptors or by a mechanism by which the broken tRNA fragments per se can have a signaling role without significantly depleting the pool of the tRNA target (Thompson and Parker 2009; Ivanov et al. 2011 and references therein). E. coli PrrC was the first example of an intracellular tRNA restriction endonuclease. The activity of PrrC is normally suppressed by its association with its cognate "antitoxin," a type I DNA restriction-modification enzyme (EcoprrI) encoded by neighboring ORFs in the prr operon (Levitz et al. 1990; Tyndall et al. 1994). tRNA ribotoxins have distinctive target specificities as follows: $E$. coli PrrC for tRNA ${ }^{\text {Lys(UUU) }}$ (Amitsur et al. 1987; Jiang et al. 2001, 2002); colicin E5 for Tyr, His, Asn, and Asp tRNAs (Ogawa et al. 1999); colicin D for tRNA ${ }^{\text {Arg }}$ (Tomita et al. 2000); enterobacterial VapC for tRNA ${ }^{\text {fmet }}$ (Winther and Gerdes 2011); K. lactis $\gamma$-toxin for tRNA ${ }^{\text {Glu(UUC) }}$ (Lu et al. 2005, 2008); and Pichia acaciae toxin for tRNA $\mathrm{th}^{\mathrm{ln}(\mathrm{UUG})}$ (Klassen et al. 2008). The findings that the cytotoxic effects of EcoPrrC, colicin E5, and colicin D are portable to budding yeast (Ogawa et al. 2009; Shigematsu et al. 2009; Meineke et al. 2011) attest that eukaryal tRNAs are vulnerable to attack by bacterial anticodon nucleases. Suppression of EcoPrrC toxicity in yeast by overexpression of tRNA ${ }^{\text {Lys(UUU) }}$ indicates that PrrC exerts toxicity via the homologous tRNA substrate in bacteria and eukarya, notwithstanding their differences in $\mathrm{tRNA}^{\text {Lys }}$ anticodon modifications.

PrrC homologs are dispersed widely among bacterial taxa, but virtually nothing is known about the biological functions and target specificities of these PrrC proteins. Nonetheless, our initial assumption was that all bacterial PrrC proteins are RNA endonucleases. Thus, it was surprising that NmePrrC, which is among the closest homologs of EcoPrrC, displayed no toxicity when expressed in yeast, even though the more distantly related SmuPrC protein was fungicidal. The lack of toxicity of NmePrrC in E. coli and the results of our domainswap experiments, in which the source of the PrrC nuclease domain emerged as the decisive factor for toxicity in yeast or E. coli, raised the prospect that either (1) NmePrrC is an active ribotoxin, but its RNA target is not present in E. coli or yeast; or (2) NmePrrC is not an active ribotoxin. With the former model in mind, we attempted to convert the nontoxic NmePrrC into a toxic derivative by screening for missense gain-of-function mutants. Remarkably, this succeeded with a single nucleotide change, of an AGG codon to a TGG codon, that replaced an Arg in NmePrrC with a Trp residue found at the equivalent position of EcoPrrC. Because the gain-of-function NmeR316W mutant exerted its toxicity via $\mathrm{tRNA}^{\mathrm{Lys}(\mathrm{UUU})}$ and because any mutation of the tryptophan 
abolished EcoPrrC toxicity, we thought we might have identified an essential species-specific determinant of PrrC target specificity.

A simpler alternative scenario was suggested to us by Gabi Kaufmann, in which NmePrrC has mutated to a nontoxic variant under selection pressure. The pressure arises because the prr operon of N. meningitidis MC58 (the source of the NmePrrC gene) has degenerated by the acquisition of premature stops and frameshifts in the NMB0831 gene that would otherwise encode the HsdS(PrrB) subunit of the restriction-modification complex that keeps E. coli PrrC in an inactive state. The Neisseria operon also has a gene encoding an IS30-family transposase inserted into the ORF that would otherwise encode the $\mathrm{HsdR}(\operatorname{PrrD})$ subunit of the restriction-modification complex. These genetic changes would seem to ablate the antitoxin that normally exerts a brake on PrrC's nuclease. In order to survive this loss, the bacterium can be expected to acquire an inactivating mutation in the PrrC anticodon nuclease. We agree with this evolutionary sequence as the likely explanation for the lack of toxicity of NmePrrC. What is remarkable is that the toxicity of $N m e \operatorname{PrC}$ can be reconstituted by a single missense change. A key lesson from these experiments is that not all PrrC homologs can be presumed to have anticodon nuclease activity. The combination of yeast toxicity assays and domain swaps affords a useful genetic strategy to assess ribotoxicity and species variations in PrrC biological activity, especially with PrrCs encoded by taxa that are not tractable genetically.

tRNA repair as an antidote to ribotoxic tRNA damage is a well-established component of the virus-host dynamic during T4 infection of $\mathrm{prr}^{+}$E. coli (Amitsur et al. 1987). We had shown previously that RNA repair enzymes can also protect yeast against growth arrest caused by K. lactis $\gamma$-toxin (Nandakumar et al. 2008). Here we extend the paradigm of RNA repair to PrrC-mediated eukaryal cytotoxicity, which can be ameliorated by either the phage T4 RNA repair system (Pnkp plus Rnl1) or plant AtRNL. In light of evidence that tRNA damage can trigger strong cellular responses, including inhibition of protein synthesis, nonlethal growth arrest, and cell death, we envision that RNA repair might play a role in tuning the severity of RNA damage or in recovering from its effects.

\section{MATERIALS AND METHODS}

\section{Yeast expression plasmids}

Yeast CEN LEU2 plasmids containing the EcoPrrC, SmuPrrC, or NmePrrC ORFs under the transcriptional control of a GAL1 promoter were described previously (Meineke et al. 2011). Domain swaps and missense mutations were introduced in the prrC genes by two-stage overlap extension PCR with fusogenic or mutagenic primers. The prrC ORF was sequenced in each case to verify the intended hybrid junctions or coding change and exclude the acquisition of unwanted coding changes during amplification and cloning. EcoRI/SalI fragments containing GAL1 $^{-}$PrrC-Ala expression cassettes were excised from the respective CEN plasmids and inserted into the multicopy yeast plasmid pRS423 ( $2 \mu$ HIS3). Yeast $2 \mu U R A 3$ plasmids bearing yeast tRNA genes were as described (Jablonowski et al. 2006; Meineke et al. 2011). RNA repair plasmids pRS423-TPI1-AtRNL $(2 \mu$ HIS3) and pRS423-TPI1-TRL1 $(2 \mu$ HIS3) carry the plant and yeast tRNA ligase genes, respectively, under the transcriptional control of the yeast TPI1 promoter. RNA repair plasmid pRS413-Pnkp/Rnl1 (CEN HIS3) vector expresses phage T4 Pnkp under the control of the yeast SLU7 promoter and T4 Rnl1 under the control of the yeast TPI1 promoter.

\section{Arabinose-inducible PrrC expression plasmids}

The EcoPrrC, SmuPrrC, NmePrrC, and chimeric PrrC open reading frames were amplified by PCR from their respective yeast CEN plasmids using a sense-strand primer that introduced an NheI site immediately $5^{\prime}$ of the translation start codon. The PCR products were digested with NheI and SalI and inserted between the corresponding restriction sites of the bacterial expression plasmid pBAD18. The prrC ORF was sequenced in each case to verify the intended coding sequence.

\section{PrrC yeast toxicity assays}

Yeast cells were transformed with PrrC plasmid DNAs, and transformants were selected on appropriate minimal synthetic media on $2 \%(\mathrm{w} / \mathrm{v})$ Bacto agar plates. Toxicity of the plasmid-encoded PrrC proteins was gauged as described (Meineke et al. 2011). Cells derived from single transformants were grown at $30^{\circ} \mathrm{C}$ in liquid culture in selective media containing $2 \%$ glucose. The cultures were adjusted to $A_{600}$ of 0.1 and then diluted in water in serial fivefold decrements. Aliquots $(3 \mu \mathrm{L})$ of the dilutions were then spotted in parallel on selective agar plates containing either $2 \%$ glucose or $2 \%$ galactose. The plates were photographed after incubation at $30^{\circ} \mathrm{C}$ for $2 \mathrm{~d}$ (glucose) or $3 \mathrm{~d}$ (galactose) unless specified otherwise.

\section{PrrC bacterial toxicity assays}

Top 10 cells ( $\operatorname{araABD}{ }^{-}$, Invitrogen) were transformed with pBADPrrC plasmids. Cells derived from single ampicillin-resistant colonies were grown in LB medium containing $200 \mu \mathrm{g} / \mathrm{mL}$ ampicillin for $4 \mathrm{~h}$ at $37^{\circ} \mathrm{C}$. The cultures were adjusted to attain $A_{600}$ of 0.025 and then diluted in 20 -fold decrements in water. Aliquots $(3 \mu \mathrm{L})$ of the dilutions were spotted in parallel on LB agar plates containing $100 \mu \mathrm{g} / \mathrm{mL}$ ampicillin with or without $0.2 \% \mathrm{~L}$-arabinose. The plates were photographed after incubation for $24 \mathrm{~h}$ at $37^{\circ} \mathrm{C}$.

\section{SUPPLEMENTAL MATERIAL}

Supplemental material is available for this article.

\section{ACKNOWLEDGMENTS}

This research was supported by NIH grant GM42498. S.S. is an American Cancer Society Research Professor.

Received August 30, 2011; accepted October 10, 2011. 


\section{REFERENCES}

Amitsur M, Levitz R, Kaufman G. 1987. Bacteriophage T4 anticodon nuclease, polynucleotide kinase, and RNA ligase reprocess the host lysine tRNA. EMBO J 6: 2499-2503.

Amitsur M, Morad I, Kaufmann G. 1989. In vitro reconstitution of anticodon nuclease from components encoded by phage $\mathrm{T} 4$ and Escherichia coli CTr5X. EMBO J 8: 2411-2415.

Amitsur M, Morad I, Chapman-Shimshoni D, Kaufmann G. 1992. HSD restriction-modification proteins partake in latent anticodon nuclease. EMBO J 11: 3129-3134.

Amitsur M, Benjamin S, Rosner R, Chapman-Shimshoni D, Meidler R, Blanga S, Kaufmann G. 2003. Bacteriophage T4-encoded Stp can be replaced as activator of anticodon nuclease by a normal host cell metabolite. Mol Microbiol 50: 129-143.

Blanga-Kanfi S, Amitsur M, Azem A, Kaufmann G. 2006. PrrCanticodon nuclease: Functional organization of a prototypical bacteria restriction RNase. Nucleic Acids Res 34: 3209-3219.

Davidov E, Kaufmann G. 2008. RloC: A wobble nucleotide-excising and zinc-responsive bacterial tRNase. Mol Microbiol 69: 1560-1574.

Ivanov P, Emara MM, Villen J, Gygi SP, Anderson P. 2011. Angiogenin-induced tRNA fragments inhibit translation initiation. Mol Cell 43: 613-623.

Jablonowski D, Schaffrath R. 2007. Zymocin, a composite chitinase and tRNase killer toxin from yeast. Biochem Soc Trans 35: 15331537.

Jablonowski D, Zink S, Mehlgarten C, Daum G, Schaffrath R. 2006. tRNA ${ }^{\text {Glu }}$ wobble uridine methylation by Trm9 identifies Elongator's key role for zymocin-induced cell death in yeast. Mol Microbiol 59: 677-688.

Jiang Y, Mediler R, Amitsur M, Kaufmann G. 2001. Specific interaction between anticodon nuclease and the tRNA ${ }^{\text {Lys }}$ wobble base. J Mol Biol 305: 377-388.

Jiang Y, Blanga S, Amitsur M, Meidler R, Krivosheyev E, Sundaram M, Bajii A, Davis DR, Kaufmann G. 2002. Structural features of $\mathrm{tRNA}^{\mathrm{Lys}}$ favored by anticodon nuclease as inferred from reactivities of anticodon stem and loop substrate analogs. J Biol Chem 277: 3836-3841.

Kaufmann G. 2000. Anticodon nucleases. Trends Biochem Sci 25: 7074.

Klaiman D, Amitsur M, Blanga-Kanfi S, Chai M, Davis DR, Kaufmann G. 2007. Parallel dimerization of a PrrC-anticodon nuclease region implicated in tRNA ${ }^{\text {Lys }}$ recognition. Nucleic Acids Res 35: 47044714.

Klassen R, Paluszynski JP, Emhoff S, Pfeiffer A, Fricke J, Meinhardt F. 2008. The primary target of the killer toxin from Pichia acaciae is tRNA $^{\text {Gln }}$. Mol Microbiol 69: 681-697.
Levitz R, Chapman D, Amitsur M, Green R, Snyder L, Kaufmann G. 1990. The optional E. coli prr locus encodes a latent form of phage T4-induced anticodon nuclease. EMBO J 9: 1383-1389.

Lu J, Huang B, Esberg A, Johanson MJO, Byström AS. 2005. The Kluyveromyces lactis $\gamma$-toxin targets tRNA anticodons. RNA 11: $1648-1654$

Lu J, Esberg A, Huang B, Byström AS. 2008. Kluyveromyces lactis $\gamma$-toxin, a ribonuclease that recognizes the anticodon stem loop of tRNA. Nucleic Acids Res 36: 1072-1080.

Meidler R, Morad I, Amitsur M, Inokuchi H, Kaufmann G. 1999. Detection of anticodon nuclease residues involved in tRNA ${ }^{\text {Lys }}$ cleavage specificity. J Mol Biol 287: 499-510.

Meineke B, Schwer B, Schaffrath R, Shuman S. 2011. Determinants of eukaryal cell killing by the bacterial ribotoxin PrrC. Nucleic Acids Res 39: 687-700.

Nandakumar J, Schwer B, Schaffrath R, Shuman S. 2008. RNA repair: An antidote to cytotoxic eukaryal RNA damage. Mol Cell 31: 278286.

Ogawa T, Tomita K, Ueda T, Watanabe K, Uozumi T, Masaki H. 1999. A cytotoxic ribonuclease targeting specific tRNA anticodons. Science 283: 2097-2100.

Ogawa T, Hidaka M, Kohno K, Masaki H. 2009. Colicin E5 ribonuclease domain cleaves Saccharomyces cerevisiae tRNAs leading to impairment of the cell growth. J Biochem 145: 461-466.

Penner M, Morad I, Snyder L, Kaufmann G. 1995. Phage T4-coded Stp: Double-edged effector of coupled DNA and tRNA-restriction systems. J Mol Biol 249: 857-868.

Shigematsu M, Ogawa T, Kido A, Kitamoto HK, Hidaka M, Masaki H. 2009. Cellular and transcriptional responses of yeast to the cleavage of cytosolic tRNAs by colicin D. Yeast 26: 663-673.

Thompson DM, Parker R. 2009. Stressing out over tRNA cleavage. Cell 138: 215-219.

Tomita K, Ogawa T, Uozumi T, Watanabe K, Masaki H. 2000. A cytotoxic ribonuclease which specifically cleaves four isoaccepting arginine tRNAs at their anticodon loops. Proc Natl Acad Sci 97: 8278-8283.

Tyndall C, Meister J, Bickle TA. 1994. The Escherichia coli prr region encodes a functional type IC DNA restriction system closely integrated with an anticodon nuclease gene. J Mol Biol 237: 266274.

Wang LK, Schwer B, Englert M, Beier H, Shuman S. 2006. Structurefunction analysis of the kinase-CPD domain of yeast tRNA ligase (Trl1) and requirements for complementation of tRNA splicing by a plant Trl1 homolog. Nucleic Acids Res 34: 517-527.

Winther KS, Gerdes K. 2011. Enteric virulence associated protein VapC inhibits translation by cleavage of initiator tRNA. Proc Natl Acad Sci 108: 7403-7407. 

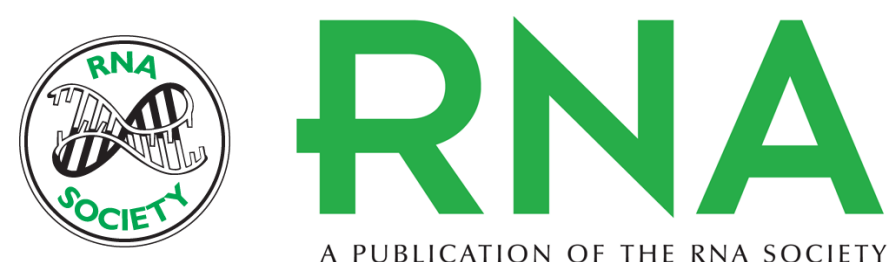

A PUBLICATION OF THE RNA SOCIETY

\section{Determinants of the cytotoxicity of PrrC anticodon nuclease and its amelioration by tRNA repair}

Birthe Meineke and Stewart Shuman

RNA 2012 18: 145-154 originally published online November 18, 2011

Access the most recent version at doi:10.1261/rna.030171.111

Supplemental http://rnajournal.cshlp.org/content/suppl/2011/11/03/rna.030171.111.DC1
Material

References This article cites 29 articles, 5 of which can be accessed free at: http://rnajournal.cshlp.org/content/18/1/145.full.html\#ref-list-1

License

Email Alerting Receive free email alerts when new articles cite this article - sign up in the box at the Service top right corner of the article or click here. 